\title{
Frontiers of Nutritional Intervention
}

\author{
Jing X. Kang \\ Massachusetts General Hospital and Harvard Medical School, Boston, Mass., USA
}

It is increasingly recognized that nutritional intervention holds great potential for the management of modern chronic disease. Understanding the relationship between nutrient metabolism and disease development is an important foundation for nutritional intervention. To maximize the utility of nutritional intervention, it is critical that we identify the factors underlying chronic disease that can be modulated by nutrients. Based on recent research, I consider the following three areas to be among the frontiers of nutritional intervention: chronic low-grade inflammation, gut microbiota, and epigenetics.

Chronic low-grade inflammation is known to be a common mechanism underlying the pathology of many chronic diseases, including obesity, diabetes, heart disease, cancer, and neurodegenerative diseases [1]. Interventions that suppress the development of chronic lowgrade inflammation could therefore be of great utility in addressing these diseases. In fact, much of the current research on the prevention and treatment of these diseases has targeted chronic inflammation. Inflammatory response involves multiple processes, including lipid mediator formation, cytokine expression, immune cell migration, etc., which can be modulated directly or indirectly by nutrients and/or their metabolites in differential manners. Certain nutrients exhibit opposing effects on inflammation. For example, omega- 6 polyunsaturated fatty acids and their metabolites generally promote inflammatory processes, while omega-3 fatty acids and their metabolites largely suppress them [2]. Thus, a full understanding of the specific nutrients' effect on chronic inflammation should be an important area of nutritional research. Given the complexity of the diet and the numerous interactions that occur between nutrients in different compositions, there remains much to be investigated about the overall effect of a diet on chronic low-grade inflammation. Future studies to elucidate how the steps of chronic low-grade inflammation are influenced by the type and quantity of nutrients and their metabolites will allow us to formulate effective interventions to modulate chronic low-grade inflammation.

The gut microbiota, which is highly variable among individuals, has recently been shown to play a key role in human health and disease [3]. More and more studies have shown that alterations in the gut microbiota can influence many physiological and pathological processes, 
including metabolism and immunity, which are associated with the development of chronic diseases. It has also been demonstrated that manipulating the gut microbiome can be an effective approach for managing certain metabolic diseases. Emerging evidence supports the notion that the diet offers the greatest accessibility to the gut microbiota and is a major factor in regulating the composition and activity of the intestinal microorganisms [4]. In fact, changes in dietary factors, including macronutrients and micronutrients, can profoundly alter the gut bacterial profile. For example, switching from a low-fat, polysaccharide-rich diet to a high-fat, high-carbohydrate Western diet can restructure the gut microbiota profile in a single day, affecting metabolism and gene expression [5]. In this context, the gut microbiota serves as an important new target for nutritional intervention. Certainly, the development of dietary means to optimize the gut microbiota is a very promising area of nutrition research. With the analysis of an individual's gut microbiota profile, tailored nutrient compositions to optimize the affected bacteria populations can serve as an interventional modality in the treatment and prevention of diseases as well as the restoration of human health. Nevertheless, much remains to be explored, as described previously [6].

Epigenetics represents another important mechanism associated with disease risk that can be modulated by environmental factors, especially the diet. Epigenetics regulates gene expression through DNA methylation, histone stability, and mRNA profiles, introducing longterm effects into the genome at any stage of life. Given that alteration of gene expression often dictates the initiation and progression of disease, regulating gene expression through epigenetics can be a critical target for disease control. Research has demonstrated that dietary patterns or lifestyle can significantly affect the epigenetic profile. For example, selenium and vitamin D status were shown to reduce the accumulation of epigenetic changes, while high blood folate increased them [7]. Epigenetics thus presents another opportunity for diet to modulate health status through the regulation of gene expression. Understanding the effects of dietary factors on epigenetics can maximize the potential of nutritional interventions.

Given the broad impact of chronic low-grade inflammation, gut microbiota, and epigenetics on chronic disease and their vulnerability to dietary factors, these three areas constitute viable targets for nutritional intervention and should be considered as the frontiers of the field. A greater understanding of the relationship between dietary factors and these areas will significantly advance the science and facilitate the practical applications of nutritional intervention for the maintenance of health as well as prevention and therapy of modern chronic diseases.

\section{References}

- 1 Ruiz-Núñez B, Pruimboom L, Dijck-Brouwer DA, Muskiet FA: Lifestyle and nutritional imbalances associated with Western diseases: causes and consequences of chronic systemic low-grade inflammation in an evolutionary context. J Nutr Biochem 2013;24:1183-1201.

2 Simopoulos AP: Omega-3 fatty acids in inflammation and autoimmune diseases. J Am Coll Nutr 2002;21:495505.

- 3 Sekirov I, Russell SL, Antunes LC, Finlay BB: Gut microbiota in health and disease. Physiol Rev 2010;90:859904.

4 Power SE, O’Toole PW, Stanton C, Ross RP, Fitzgerald GF: Intestinal microbiota, diet and health. Br J Nutr 2014; 111:387-402.

5 Turnbaugh PJ, Ridaura VK, Faith JJ, Rey FE, Knight R, Gordon JI: The effect of diet on the human gut microbiome: a metagenomic analysis in humanized gnotobiotic mice. Sci Transl Med 2009;1:6ra14.

6 Kang JX: Gut microbiota and personalized nutrition. J Nutrigenet Nutrigenomics 2013;6:1-2.

- 7 Tapp HS, Commane DM, Bradburn DM, Arasaradnam R, Mathers JC, Johnson IT, Belshaw NJ: Nutritional factors and gender influence age-related DNA methylation in the human rectal mucosa. Aging Cell 2012;12:148-155. 